\title{
ParaHaplo 3.0: A program package for imputation and a haplotype-based whole-genome association study using hybrid parallel computing
}

\author{
Kazuharu Misawa ${ }^{1 *}$ and Naoyuki Kamatani
}

\begin{abstract}
Background: Use of missing genotype imputations and haplotype reconstructions are valuable in genome-wide association studies (GWASs). By modeling the patterns of linkage disequilibrium in a reference panel, genotypes not directly measured in the study samples can be imputed and used for GWASs. Since millions of single nucleotide polymorphisms need to be imputed in a GWAS, faster methods for genotype imputation and haplotype reconstruction are required.

Results: We developed a program package for parallel computation of genotype imputation and haplotype reconstruction. Our program package, ParaHaplo 3.0, is intended for use in workstation clusters using the Intel Message Passing Interface. We compared the performance of ParaHaplo 3.0 on the Japanese in Tokyo, Japan and Han Chinese in Beijing, and Chinese in the HapMap dataset. A parallel version of ParaHaplo 3.0 can conduct genotype imputation 20 times faster than a non-parallel version of ParaHaplo.

Conclusions: ParaHaplo 3.0 is an invaluable tool for conducting haplotype-based GWASs. The need for faster genotype imputation and haplotype reconstruction using parallel computing will become increasingly important as the data sizes of such projects continue to increase. ParaHaplo executable binaries and program sources are available at http://en.sourceforge.jp/projects/parallelgwas/releases/.
\end{abstract}

Keywords: ParaHaplo, haplotype reconstruction, genotype imputation, parallel computing, HapMap, GWAS

\section{Background}

Recent advances in various high-throughput genotyping technologies have allowed us to test allelic frequency differences between case and control populations on a genome-wide scale [1]. Genome-wide association studies (GWASs) are used to compare the frequency of alleles or genotypes of a particular variant between cases and controls for a particular disease across a given genome [2-4]. More than a million single nucleotide polymorphisms (SNPs) are analyzed in SNP-based GWASs and haplotype-based GWASs [5,6].

By modeling the patterns of linkage disequilibrium in a reference panel, genotypes not directly measured in

\footnotetext{
* Correspondence: kazumisawa@riken.jp

'Research Program for Computational Science, Research and Development Group for Next-Generation Integrated Living Matter Simulation, and Fusion of Data and Analysis Research and Development Team, RIKEN, 4-6-1

Shirokane-dai, Minato-ku, Tokyo 108-8639, Japan

Full list of author information is available at the end of the article
}

the study samples can be imputed [7]. SNP genotype imputation has been proposed as a powerful means to include genetic markers into large-scale disease association studies without the need to actually genotype them $[8,9]$

To quickly conduct GWASs, we developed a software package for the parallel computation of genotype imputation and haplotype reconstruction called ParaHaplo 3.0. ParaHaplo 3.0 contains all of the functions of ParaHaplo 1.0 [5] and ParaHaplo 2.0 [6], plus it can conduct genotype imputation and haplotype reconstruction using MACH 1.0 [10]. ParaHaplo 3.0 is based on the principle of data parallelism, a programming technique used to split large datasets into smaller ones that can be run in a parallel concurrent fashion [11]. ParaHaplo 3.0 is intended for use in workstation clusters using the Intel Message Passing Interface (MPI).

\section{Biomed Central}


Using ParaHaplo 3.0, we estimated haplotypes using the genotype data of the Japanese from Tokyo (JPT) and the Han Chinese from Beijing (CHB) obtained from the HapMap dataset $[12,13]$. Using ParaHaplo 3.0, we compared the speed of haplotype estimation using parallel computation to the number of processors.

\section{Methods}

\section{Software overview}

ParaHaplo supports the genotype data in the HapMap format [14] and the BioBank Japan format [15]. ParaHaplo 3.0 requires an input file of haplotype block boundaries. ParaHaplo 3.0 can conduct genotype imputation and haplotype reconstruction using MACH 1.0 [10]. ParaHaplo 3.0 can also conduct haplotype estimation using PHASE 2.1 [16] and SNPHAP 1.3.1 [17] algorithms. By using hybrid MPI + OpenMP parallelization [18], ParaHaplo 3.0 can conduct haplotype-based GWAS faster than previous versions.

\section{Parallel computing using MPI methods}

ParaHaplo 3.0 is implemented in an MPI-C multithreaded package. The MPI package allows us to construct parallel computing programs on multiprocessors. The genome-wide polymorphism data is broken down into user-defined haplotype blocks, and the MPI Bcast function is used to distribute a single block of haplotype data into each processor. Each processor executes Mach 1.0 [10] and conducts genotype imputation and haplotype reconstruction of a single linkage disequilibrium (LD) block. Once the haplotypes of each LD block are completely estimated, the results are compiled into a single genome-wide dataset through use of the MPIGatherv function. ParaHaplo 3.0 is compatible with
OpenMPI 1.2.5 and MPICH 1.2.7p1. Users can compile the source code using a GCC compiler, an Intel C compiler, or a Fujitsu $\mathrm{C}$ compiler, so that Haplotype-based GWAS can be run on Linux-based PC clusters as well as on K computer (http://www.fujitsu.com/global/news/ pr/archives/month/2009/20090717-01.html).

\section{Hardware}

A PC cluster at RIKEN Integrated Cluster of Clusters (RICC) was used when the computational time was measured. The program was compiled using an Intel $\mathrm{C}$ compiler. The numbers of processing units used included 1, 2, 4, 8, 16, 32, and 64 .

\section{Example data}

An example GWAS is presented here. We used ParaHaplo 3.0 to compare genome-wide genotype data of JPT and CHB from HapMap 3.0 [13]. Some individuals were excluded because they contain too many untyped SNPs. As the reference panel, we used 20,086 SNPs of 170 people. JPT data set was used to be imputed. JPT data set consisted of 82 people with 2,392 SNPs being untyped. Haplotype blocks were obtained as LD blocks using the method outlined by Gabriel et al. [19] and the Haploview program [20]. The entire JPT and CHB genomes were divided into 106,149 haplotype blocks by Haploview [20]. Among them, 1,536 haplotype blocks were on chromosome 22.

\section{Results and discussion}

\section{Genotype Imputation and Haplotype Reconstruction of JPT and CHB}

Figure 1 shows the input and the output data of haplotype phasing. Each line corresponds to a SNP site.

\begin{tabular}{|c|c|c|c|c|c|c|c|c|c|c|c|c|c|c|c|c|c|}
\hline rs\# & alleles & chrom & \multicolumn{2}{|c|}{ pos } & strand & assembly\# & center & \multicolumn{2}{|c|}{ protLSID } & \multicolumn{2}{|c|}{ assayLSID } & \multicolumn{2}{|c|}{ panelLSID } & QCcode & NA18942 & NA18940 & NA18945 \\
\hline rs9617528 & $\mathrm{C} / \mathrm{T}$ & chr22 & \multicolumn{2}{|c|}{14441016} & + & ncbi_b36 & bbs & & $\mathrm{x}$ & \multicolumn{2}{|c|}{$\mathrm{y}$} & \multicolumn{2}{|c|}{$\mathrm{z}$} & $\mathrm{QC}+$ & TT & TT & TT \\
\hline rs8138488 & $\mathrm{C} / \mathrm{T}$ & chr22 & \multicolumn{2}{|c|}{14870204} & + & ncbi_b36 & bbs & & $\mathrm{x}$ & \multicolumn{2}{|c|}{$\mathrm{y}$} & \multicolumn{2}{|l|}{$\mathrm{z}$} & QC+ & $\mathrm{CT}$ & TT & TT \\
\hline rs9617160 & $\mathrm{C} / \mathrm{T}$ & chr22 & \multicolumn{2}{|c|}{14871206} & + & ncbi_b36 & bbs & & $\mathrm{x}$ & \multicolumn{2}{|c|}{$\mathrm{y}$} & \multicolumn{2}{|l|}{$\mathrm{z}$} & $\mathrm{QC}+$ & $\mathrm{CC}$ & $\mathrm{CC}$ & $\mathrm{CC}$ \\
\hline \multicolumn{18}{|c|}{ b. Reference panel (partial) } \\
\hline \begin{tabular}{|r|}
$\mathrm{rs} \#$ \\
\end{tabular} & \multicolumn{3}{|c|}{ 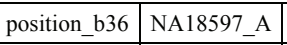 } & \multicolumn{2}{|c|}{ NA18597_B } & NA18615_A & \multicolumn{2}{|c|}{ NA18615_B } & \multicolumn{2}{|c|}{ NA18557_A } & \multicolumn{2}{|c|}{\begin{tabular}{|l|l} 
NA18557_B & I \\
\end{tabular}} & \multicolumn{2}{|c|}{ NA18628_A } & NA18628_B & & \\
\hline rs4819391 & 14550436 & \multicolumn{2}{|c|}{$\mathrm{A}$} & \multicolumn{2}{|c|}{$\mathrm{A}$} & A & \multicolumn{2}{|c|}{$\mathrm{A}$} & \multicolumn{2}{|c|}{ A } & \multicolumn{2}{|c|}{$\mathrm{A}$} & \multicolumn{2}{|c|}{$\mathrm{A}$} & $\mathrm{A}$ & & \\
\hline rs11089128 & 145602 & & $\mathrm{~A}$ & & G & A & A & & A & & & G & & A & G & & \\
\hline rs11912265 & 147155 & & A & & A & A & A & & A & & & A & & A & A & & \\
\hline c. Result of & imputation & (partial) & & & & & & & & & & & & & & & \\
\hline \begin{tabular}{|l|}
$\mathrm{rs} \#$ \\
\end{tabular} & alleles & chrom & po & & strand & assembly\# & center & prot & tLSID & assayL & SID & panelLSI & & QCcode & NA18942 & NA18940 & NA18945 \\
\hline rs4819391 & A & $\operatorname{chr} 22$ & 14550 & 436 & + & 0 & 0 & & 0 & 0 & & 0 & & 0 & $\mathrm{AA}$ & $\mathrm{AA}$ & AA \\
\hline rs11089128 & $\mathrm{A} / \mathrm{G}$ & $\operatorname{chr} 22$ & 14560 & 203 & + & 0 & 0 & & 0 & 0 & & 0 & & 0 & AA & $\mathrm{AA}$ & $\mathrm{AG}$ \\
\hline rs11912265 & $\mathrm{A}$ & chr22 & 14715 & 506 & + & 0 & 0 & & 0 & 0 & & 0 & & 0 & AA & $\mathrm{AA}$ & $\mathrm{AA}$ \\
\hline
\end{tabular}

Figure 1 Results of genotype imputation by ParaHaplo 3.0. a. Input data to be imputed. From the $1^{\text {st }}$ to $11^{\text {th }}$ columns are the same as HapMap data format. b. Reference panel. Reference panel must be phased. c. Imputed data. From the $1^{\text {st }}$ to $11^{\text {th }}$ columns are the same as HapMap data format. 
From the $1^{\text {st }}$ to $11^{\text {th }}$ columns are the same as HapMap data format. For example, the $1^{\text {st }}$ column shows the rs number of each SNP. From the $12^{\text {th }}$ column to the end of the line, each column corresponds to the genotype at the SNP site of one individual. Figure 1a shows an example of the input data to be imputed. From the $1^{\text {st }}$ to $11^{\text {th }}$ columns are the same as HapMap data format. Figure $1 \mathrm{~b}$ shows reference panel. Reference panel must be phased by using ParaHaplo 2.0 [6] or by other programs. Figure 1c shows the result of imputation. From the $1^{\text {st }}$ to $11^{\text {th }}$ columns are the same as HapMap data format. Columns without any information are filled by 0 .

\section{Calculation Time}

Table 1 shows the elapsed times and the speedups associated with the use of ParaHaplo 3.0 using the genotype data of chromosome 22 for haplotype estimation. The speedup ratio is the ratio of the computation time of a single processor to that of multiple processors. As shown in Table 1 the calculation time decreased as the number of processors increased. When 64 processors were used, ParaHaplo functioned 20 times faster than the non-parallel program.

\section{Parallel Computation of Haplotype-Based GWAS}

The results show that the parallel computing ability of ParaHaplo 3.0 for haplotype estimation was 20 times faster than that of the non-parallel version of ParaHaplo 3.0. In this study, we used a total of $89 \mathrm{JPT}$ and $\mathrm{CHB}$ individuals whose genotypes had been determined during the HapMap project [12]. When a single processor was used, haplotype estimation for chromosome 22 took more than $4 \mathrm{~h}$; if 9,000 individuals were to be analyzed under the same conditions, the analysis would take more than 2 weeks. However, if ParaHaplo 3.0 was used on a workstation with 64 processors, the same analysis would take less than 1 day. However, the relatively lower speed ratio could be affected by the inflation of sample size [11]. Further study is required.

Table 1 Elapsed times and speedups obtained using ParaHaplo 3.0 in the imputation process

\begin{tabular}{ccccc}
\hline Number of Processing Units & \multicolumn{3}{c}{ Elapsed Time } & Speed Ratio $^{\mathbf{a}}$ \\
\hline 1 & $4 \mathrm{~h}$ & $21 \mathrm{~m}$ & $59 \mathrm{~s}$ & 1 \\
2 & $2 \mathrm{~h}$ & $12 \mathrm{~m}$ & $2 \mathrm{~s}$ & 1.98 \\
4 & $1 \mathrm{~h}$ & $10 \mathrm{~m}$ & $3 \mathrm{~s}$ & 3.74 \\
8 & & $38 \mathrm{~m}$ & $46 \mathrm{~s}$ & 6.76 \\
16 & & $24 \mathrm{~m}$ & $30 \mathrm{~s}$ & 10.69 \\
32 & & $13 \mathrm{~m}$ & $25 \mathrm{~s}$ & 19.51 \\
64 & & $11 \mathrm{~m}$ & $25 \mathrm{~s}$ & 22.94 \\
\hline
\end{tabular}

${ }^{a}$ Ratio of computation time of a single processor to computation time of multiple processors
Even when 64 processors were used, the speedup ratio was only 22 because of the variations in the LD block size. ParaHaplo is based on data parallelism, and our result showed that the computation time of each genotype imputation was approximately proportional to the number of SNPs within the LD block (data not shown); therefore, we believe that a large LD block may create a computational bottleneck as does in haplotype estimation [6].

\section{Conclusions}

We developed ParaHaplo 3.0, a set of computer programs, for the parallel computation of haplotype estimation and accurate P values in haplotype-based GWASs. ParaHaplo is intended for use in workstation clusters using the Intel MPI. Using ParaHaplo, we conducted haplotype estimation of JPT and $\mathrm{CHB}$ genotype data taken from the HapMap 3.0 dataset [12].

These results indicate that when the number of processors is sufficient, the parallel computing abilities of ParaHaplo are 20 times faster than those of non-parallel programs. Accurate and complete genotypes have been obtained for more than a million SNPs [15], and $>10,000$ individuals are now being genotyped [21]. The need for fast haplotype estimation using parallel computing will become increasingly important as project data sizes continue to increase.

\section{Availability and Requirements}

-Project name: ParaHaplo 3.0

-Project home page: http://sourceforge.jp/projects/ parallelgwas/releases/46982

-Operating systems: Platform independent

- Programming language: Java and C

-Other requirements: OpenMPI version 1.2.5, or $\mathrm{MPICH}$ version 1.2.7p1

-License: MIT license

-Any restrictions for use by non-academics: License required

List of abbreviations

GWAS, Genome-Wide Association Study; SNP, Single Nucleotide

Polymorphism; LD, Linkage Disequilibrium; RAT, Rapid Association Test; SPT, Standard Permutation Test; MCMC, Markov-chain Monte Carlo; JPT, Japanese Tokyo; CHB, Han Chinese Beijing

\section{Acknowledgements}

This study was supported by the "Next-Generation Integrated Living Matter Simulation," a national project of the Ministry of Education, Culture, Sports, Science and Technology (MEXT), and by the Research Project for Personalized Medicine (MEXT).

\section{Author details}

${ }^{1}$ Research Program for Computational Science, Research and Development Group for Next-Generation Integrated Living Matter Simulation, and Fusion of Data and Analysis Research and Development Team, RIKEN, 4-6-1 Shirokane-dai, Minato-ku, Tokyo 108-8639, Japan. ${ }^{2}$ Laboratory for Statistical Analysis, RIKEN Center for Genomic Medicine, Tokyo, Japan. 


\section{Authors' contributions}

KM designed the software and wrote the manuscript, and NK supervised the project. Both authors have read and approved the final manuscript.

\section{Competing interests}

The authors declare that they have no competing interests.

Received: 28 February 2011 Accepted: 24 May 2011

Published: 24 May 2011

\section{References}

1. Hirschhorn JN, Daly MJ: Genome-wide association studies for common diseases and complex traits. Nat Rev Genet 2005, 6(2):95-108.

2. Ozaki K, Ohnishi Y, lida A, Sekine A, Yamada R, Tsunoda T, Sato H, Hori M, Nakamura Y, Tanaka T: Functional SNPs in the lymphotoxin-alpha gene that are associated with susceptibility to myocardial infarction. Nat Genet 2002, 32(4):650-654.

3. Onouchi Y, Gunji T, Burns JC, Shimizu C, Newburger JW, Yashiro M, Nakamura Y, Yanagawa H, Wakui K, Fukushima Y, Kishi F, Hamamoto K, Terai M, Sato Y, Ouchi K, Saji T, Nariai A, Kaburagi Y, Yoshikawa T, Suzuki K, Tanaka T, Nagai T, Cho H, Fujino A, Sekine A, Nakamichi R, Tsunoda T, Kawasaki T, Hata A: ITPKC functional polymorphism associated with Kawasaki disease susceptibility and formation of coronary artery aneurysms. Nat Genet 2008, 40(1):35-42.

4. Tokuhiro S, Yamada R, Chang X, Suzuki A, Kochi Y, Sawada T, Suzuki M, Nagasaki M, Ohtsuki M, Ono M, Furukawa H, Nagashima M, Yoshino S, Mabuchi A, Sekine A, Saito S, Takahashi A, Tsunoda T, Nakamura Y, Yamamoto K: An intronic SNP in a RUNX1 binding site of SLC22A4, encoding an organic cation transporter, is associated with rheumatoid arthritis. Nat Genet 2003, 35(4):341-348.

5. Misawa K, Kamatani N: ParaHaplo: A program package for haplotypebased whole-genome association study using parallel computing. Source Code Biol Med 2009, 4(1):7.

6. Misawa K, Kamatani N: ParaHaplo 2.0: a program package for haplotypeestimation and haplotype-based whole-genome association study using parallel computing. Source Code Biol Med 2010, 5(1):5.

7. Li Y, Willer C, Sanna S, Abecasis G: Genotype imputation. Annu Rev Genomics Hum Genet 2009, 10:387-406.

8. Marchini J, Howie B, Myers S, McVean G, Donnelly P: A new multipoint method for genome-wide association studies by imputation of genotypes. Nat Genet 2007, 39(7):906-913.

9. Nothnagel M, Ellinghaus D, Schreiber S, Krawczak M, Franke A: A comprehensive evaluation of SNP genotype imputation. Hum Genet 2009, 125(2):163-171.

10. Li Y, Abecasis GR: Mach 1.0: rapid haplotype reconstruction and missing genotype inference. Am J Hum Genet 2006, 79:S2290.

11. Culler DE, Gupta A, Singh JP: Parallel Computer Architecture: A Hardware/ Software Approach. San Francisco, CA: Morgan Kaufmann Publishers; 1997.

12. The International HapMap Consortium: The International HapMap Project. Nature 2003, 426(6968):789-796.

13. Altshuler DM, Gibbs RA, Peltonen L, Dermitzakis E, Schaffner SF, Yu F, Bonnen PE, de Bakker PI, Deloukas P, Gabriel SB, Gwilliam R, Hunt S, Inouye M, Jia X, Palotie A, Parkin M, Whittaker P, Chang K, Hawes A, Lewis LR, Ren Y, Wheeler D, Muzny DM, Barnes C, Darvishi K, Hurles M, Korn JM, Kristiansson K, Lee C, McCarrol SA, et al: Integrating common and rare genetic variation in diverse human populations. Nature 2010, 467(7311):52-58

14. Marchini J, Cutler D, Patterson N, Stephens M, Eskin E, Halperin E, Lin S, Qin ZS, Munro HM, Abecasis GR, Donnelly P: A comparison of phasing algorithms for trios and unrelated individuals. Am J Hum Genet 2006, 78(3):437-450.

15. Nakamura Y: The BioBank Japan Project. Clin Adv Hematol Oncol 2007, 5(9):696-697.

16. Mardanov AV, Ravin NV, Kuznetsov BB, Samigullin TH, Antonov AS, Kolganova TV, Skyabin KG: Complete Sequence of the Duckweed (Lemna minor) Chloroplast Genome: Structural Organization and Phylogenetic Relationships to Other Angiosperms. J Mol Evol 2008.

17. SNPHAP - A program for estimating frequencies of large haplotypes of SNPs. [http://www-gene.cimr.cam.ac.uk/clayton/software/].
18. Rabenseifner R: Hybrid parallel programming on HPC platforms. The proceedings of the Fifth European Workshop on OpenMP, EWOMP 2003; Aachen, Germany 2003.

19. Gabriel SB, Schaffner SF, Nguyen H, Moore JM, Roy J, Blumenstiel B, Higgins J, DeFelice M, Lochner A, Faggart M, Liu-Cordero SN, Rotimi C, Adeyemo A, Cooper R, Ward R, Lander ES, Daly MJ, Altshuler D: The structure of haplotype blocks in the human genome. Science 2002, 296(5576):2225-2229.

20. Barrett JC, Fry B, Maller J, Daly MJ: Haploview: analysis and visualization of LD and haplotype maps. Bioinformatics 2005, 21(2):263-265.

21. Kamatani Y, Matsuda K, Okada Y, Kubo M, Hosono N, Daigo Y, Nakamura Y, Kamatani N: Genome-wide association study of hematological and biochemical traits in a Japanese population. Nat Genet 2010, 42(3):210-215.

doi:10.1186/1751-0473-6-10

Cite this article as: Misawa and Kamatani: ParaHaplo 3.0: A program package for imputation and a haplotype-based whole-genome association study using hybrid parallel computing. Source Code for Biology and Medicine 2011 6:10.

\section{Submit your next manuscript to BioMed Central and take full advantage of:}

- Convenient online submission

- Thorough peer review

- No space constraints or color figure charges

- Immediate publication on acceptance

- Inclusion in PubMed, CAS, Scopus and Google Scholar

- Research which is freely available for redistribution 